\title{
Changing Tax Rates Impacts on the Risk Level of Vietnam Construction Material Firms
}

\author{
Dinh Tran Ngoc Huy* \\ Banking University, HCMC-GSIM, International University of Japan, Japan
}

\begin{abstract}
The Viet Nam stock market has been affected by the financial crisis 2007-2009. This study analyzes the impacts of tax policy on market risk for the listed firms in the construction material industry as it becomes necessary.

First, by using quantitative and analytical methods to estimate asset and equity beta of total 57 listed companies in Viet Nam construction material industry with a proper traditional model, we found out that the beta values, in general, for many companies are acceptable.

Second, under 3 different scenarios of changing tax rates $(20 \%, 25 \%$ and $28 \%)$, we recognized that there is not large disperse in equity beta values, estimated at 0,957, 1,011 and 0,961. These values are lower than those of the listed VN construction firms.

Third, by changing tax rates in 3 scenarios $(25 \%, 20 \%$ and $28 \%)$, we recognized equity /asset beta mean and asset beta var increase if tax rate increases from $20 \%$ to $25 \%$, then decrease when tax rate goes up from $25 \%$ to $28 \%$.

Finally, this paper provides some outcomes that could provide companies and government more evidence in establishing their policies in governance.
\end{abstract}

Keywords: Equity beta; Financial structure; Financial crisis; Risk; Tax rate; Construction material industry; Jell classification

\section{Introduction}

During the global crisis 2007-2009, Viet Nam stock market (Figure 1) has difficulties and opportunities. In this study, we perform a market risk analysis with quantitative methodology based on asset and equity beta of 57 listed companies in the group of construction material firms. The above industry faced many difficulties in previous years such as how to increase the number of customers, service quality and revenues; now, they have to deal with some problems from the global crisis. From 2009-2011, the local government and central bank have performed some effective macro policies including fiscal policy (tax) to help the economy to recover.

Together with the development of real estate industry, throughout many recent years, Viet Nam construction industry is considered as one of active economic sectors, which has certain positive effect for the economy. Hence, this paper emphasizes on analyzing un-diversifiable risk in the above industry in one of emerging markets: Vietnam stock market (Figure 2) during and after the financial crisis 2007-2009. There is no research, so far, done on the same topic.

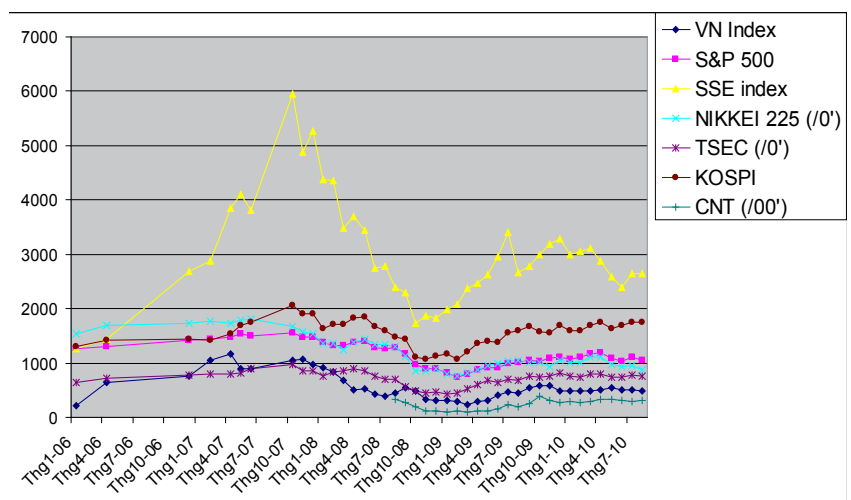

Figure 1: VNI Index and other stock market index during crisis 2006-2010.
This paper is organized as follow. The research issues and literature review will be covered in next sessions 2 and 3, for a short summary. Then, methodology and conceptual theories are introduced in session 4 and 5. Session 6 describes the data in empirical analysis. Session 7 presents empirical results and findings. Next, session 8 covers the analytical results. Then, session 9 presents analysis of risk. Lastly, session 10 will conclude with some policy suggestions. This paper also supports readers with references, exhibits and relevant web sources.

\section{Research Issues}

We mention some issues on the estimating of impacts of tax rates on beta for listed construction companies in Viet Nam stock exchange as following:

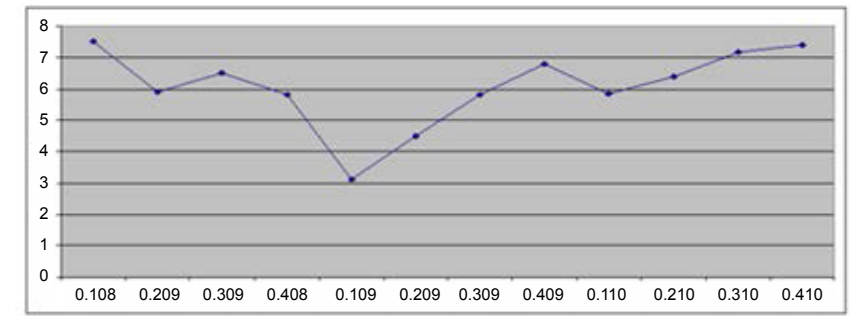

Figure 2: GDP growth Việt Nam 2006-2010 (source: Bureau Statistic).

*Corresponding author: Dinh Tran Ngoc Huy, Banking University, HCMCGSIM, International University of Japan, Japan, Tel: 0908.43.1117; 0934.814622; 08.39934160; E-mail: dtnhuy2010@gmail.com

Receved November 08, 2013; Accepted February 10, 2014; Published February 17,2014

Citation: Huy DTN (2014) Changing Tax Rates Impacts on the Risk Level of Vietnam Construction Material Firms. J Entrepren Organiz Manag 3: 110. doi: 10.4172/2169-026X.1000110

Copyright: (c) 2014 Huy DTN. This is an open-access article distributed under the terms of the Creative Commons Attribution License, which permits unrestricted use, distribution, and reproduction in any medium, provided the original author and source are credited. 


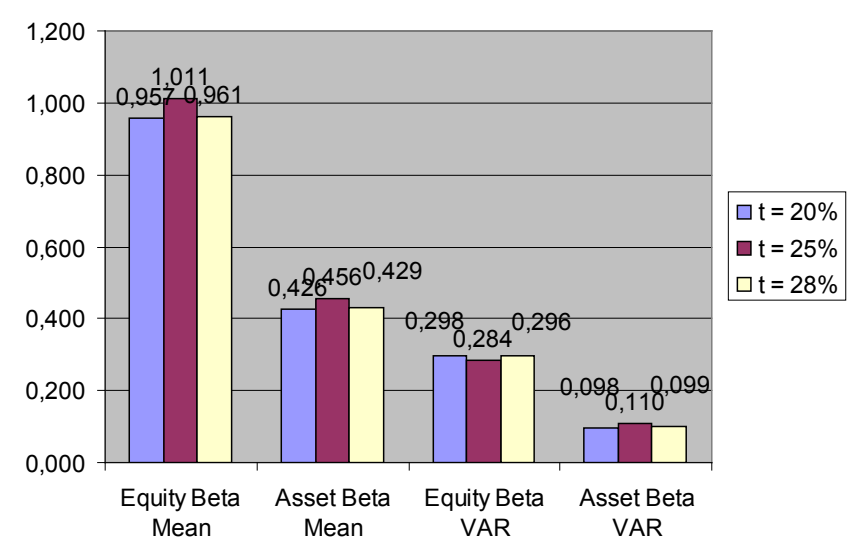

Figure 3: Comparing statistical results of three (3) scenarios of changing tax rate.

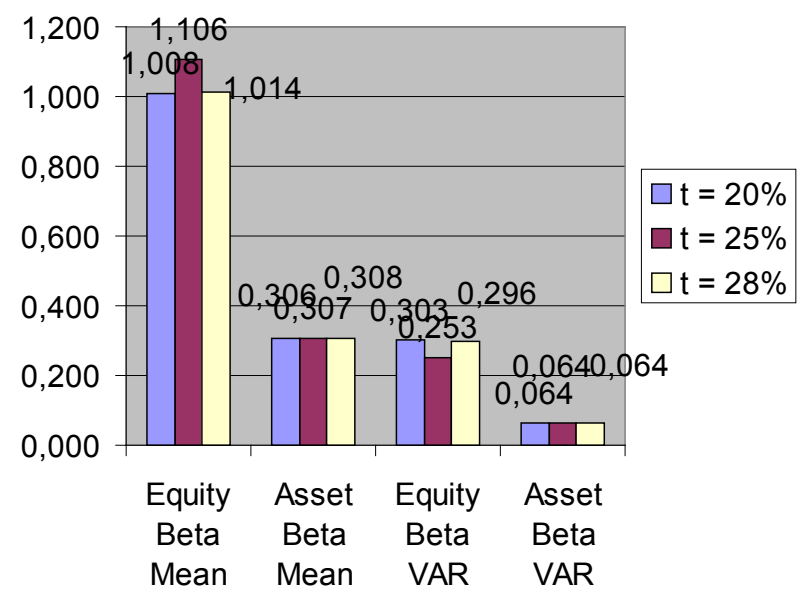

Figure 4: Comparing statistical results of three (3) scenarios of changing tax rate of 104 listed construction firms.

Issue 1: Whether the risk level of construction firms under the different changing scenarios of tax rates increase or decrease so much.

Issue 2: Whether the disperse distribution of beta values become large in the different changing scenarios of tax rates estimated in the construction industry (Figures 3 and 4).

\section{Literature review}

Smith [1] mentions in Chicago, properties located in a designated TIF (tax increment financing) district will exhibit higher rates of appreciation after the area is designated a qualifying TIF district when compared to those properties selling outside TIF districts, and when compared to properties that sell within TIF district boundaries prior to designation.

Then, Vikas and David [2] said that the change in the tax law, by itself did not because the housing bubble but other factors play a larger role, such as: a failure by regulators to intervene.

Vieri [3,4] found out tax system could affect economic factors that trigger the financial crisis, such as: mortgage interest, management performance - based remuneration, and securitization process. Thomas [5] recognized that tax incentives may indeed have exacerbated the behavior of economic agents, leading them to wrong economic decision. George and Yau [6] found that there is a positive relationship between transaction cost and price volatility, suggesting that the imposition of a transaction tax could increase financial market fragility, increasing the likelihood of a financial crisis rather than reducing it.

Next, Rudd [7] said that greater tax bias is associated with significantly higher aggregate bank leverage, and this in turn is associated with a significantly greater chance of crisis.

Then, Sung et al. [8] also indicated that business property values are more responsive to changes in tax rates as compared to residential property.

Finally, tax rate can be considered as one among many factors that affect business risk of construction material firms.

\section{Conceptual Theories}

\section{The impact of fiscal policy on the economy}

Tax policy is one among major fiscal policies. In a specific industry such as construction material industry, on the one hand, using tax policy with a decrease or increase in tax rate could affect tax revenues, profit after tax, demand, the allocation of economic resources and financial results and compensation and jobs of the industry. Beside, using tax policies and rules could affect the excessive using of leverage or debt, as well as the number of financial transactions in businesses and the economy.

In the financial crisis 2007-2009, tax policy is not the cause whereas the government could use it to help the economy and business. However, we might note that the wrong use of tax policy might partly cause the crisis impacts become more serious.

\section{Methodology}

The impacts from the financial crisis happened during the period 2007-201; hence, in this study, we use the live data from the stock exchange market in Viet Nam (HOSE and HNX) during the four or five years period to estimate systemic risk results and tax impacts.

In this research, analytical research method is used, philosophical method is used and specially, tax rate scenario analysis method is used. Analytical data is from the situation of listed construction material firms in VN stock exchange and current tax rate is $25 \%$.

Finally, we use the results to suggest policy for both these enterprises, relevant organizations and government.

\section{General Data Analysis}

The research sample has 57 listed firms in the construction material market with the live data from the stock exchange.

Firstly, we estimate equity beta values of these firms and use financial leverage to estimate asset beta values of them. Secondly, we change the tax rate from $25 \%$ to $28 \%$ and $20 \%$ to see the sensitivity of beta values. In 3 cases (rate $=20 \%, 25 \%$, and $28 \%$ ), asset beta mean is estimated at 0,426, 0,456 and 0,429. Also in 3 scenarios, we find out var of asset beta estimated at 0,098, 0,110 and 0,099 (almost the same) which shows small risk dispersion. Tax rate changes almost have no effect on asset beta var under financial leverage.

\section{Empirical Research Findings and Discussion}

In the below section, data used are from total 57 listed construction 
Citation: Huy DTN (2014) Changing Tax Rates Impacts on the Risk Level of Vietnam Construction Material Firms. J Entrepren Organiz Manag 3: 110. doi: $10.4172 / 2169-026 X .1000110$

Page 3 of 9

material companies on VN stock exchange (HOSE and HNX mainly). In the scenario 1 , current tax rate is $25 \%$ which is used to calculate market risk (beta). Then, two (2) tax rate scenarios are changed up to $28 \%$ and down to $20 \%$, compared to the current corporate tax rate (Tables 1-7).
Market risk (beta) under the impact of tax rate, includes: 1) equity beta; and 2) asset beta.

- Scenario 1: current tax rate is $25 \%$

In the case of tax rate of $25 \%$, all beta values of 57 listed firms on

\begin{tabular}{|c|c|c|c|}
\hline Year & Borrowing Interest rates & Deposit Rates & Note \\
\hline 2011 & $18 \%-22 \%$ & $13 \%-14 \%$ & \\
\hline 2010 & $19 \%-20 \%$ & $13 \%-14 \%$ & \multirow{4}{*}{$\begin{array}{c}\text { Approximately } \\
\text { (2007: required reserves ratio at SBV is } \\
\text { changed from } 5 \% \text { to } 10 \% \text { ) } \\
\text { (2009: special supporting interest rate is } 4 \% \text { ) }\end{array}$} \\
\hline 2009 & $9 \%-12 \%$ & $9 \%-10 \%$ & \\
\hline 2008 & $19 \%-21 \%$ & $15 \%-16,5 \%$ & \\
\hline 2007 & $12 \%-15 \%$ & $9 \%-11 \%$ & \\
\hline
\end{tabular}

Table 1: Interest rates in banking industry during crisis(source: Viet Nam commercial banks).

\begin{tabular}{|c|c|}
\hline Year & Basic rate \\
\hline 2011 & $9 \%$ \\
\hline 2010 & $8 \%$ \\
\hline 2009 & $7 \%$ \\
\hline 2008 & $8,75 \%-14 \%$ \\
\hline 2007 & $8,25 \%$ \\
\hline 2006 & $8,25 \%$ \\
\hline 2004 & $7,8 \%$ \\
\hline 2003 & $7,5 \%$ \\
\hline 2002 & $7,5 \%$ \\
\hline 2000 & $7,44 \%$ \\
\hline
\end{tabular}

Table 2: Basic interest rate changes in Viet Nam(source: State Bank of Viet Nam and Viet Nam economy).

\begin{tabular}{|c|c|c|c|}
\hline Year & Inflation & GDP & USD/VND rate \\
\hline 2011 & $18 \%$ & $5,89 \%$ & 20.670 \\
\hline 2010 & $11,75 \%$ (Estimated at Dec 2010) & $6,5 \%$ (expected) & 19.495 \\
\hline 2009 & $6,88 \%$ & $5,2 \%$ & 17.000 \\
\hline 2008 & $22 \%$ & $6,23 \%$ & 17.700 \\
\hline 2007 & $12,63 \%$ & $8,44 \%$ & 16.132 \\
\hline 2006 & $6,6 \%$ & $8,17 \%$ & \\
\hline 2005 & $8,4 \%$ & & \\
\hline Note & \multicolumn{3}{|c|}{ approximately } \\
\hline
\end{tabular}

Table 3: Inflation, GDP growth and macroeconomics factors(source: Viet Nam commercial banks and economic statistical bureau).

\begin{tabular}{|c|c|c|c|c|c|}
\hline Order & Rank & Company name & CEO & Tax code & Industry \\
\hline 1 & 1 & CÔNG TY CP LÂM SẢN PISICO QUẢNG NAM & Phan Văn Cường & 4000405162 & Kinh doanh nông, lâm sản \\
\hline 2 & 2 & CÔNG TY CP VÀNG BAC ĐÁ QUÝ SJC CÀN THƠ & Lê Phát Vinh & 1800636290 & Vàng, bac, đá quý \\
\hline 3 & 3 & CÔNG TY CP SƠI PHÚ NAM & Hoàng Văn Thám & 3300491474 & Sản xuất sơii, dêt \\
\hline 4 & 4 & CÔNG TY CP VÀNG BAC ĐÁ QUÝ BÉN THÀNH & Tề Trí Dũng & 300847936 & Vàng, bac, đá quý \\
\hline 5 & 5 & CÔNG TY CP MAY CHIÉN THÁNG & Ninh Thị Ty & 100101058 & Ngành may \\
\hline 6 & 6 & CÔNG TY TNHH DU LICH TRÀN VIẸT & Nguyễn Mạnh Cương & 301069809 & Du lich, khách san \\
\hline 7 & 7 & N.A & & 2012701 & \\
\hline 8 & 8 & CÔNG TY TNHH TRUYĖN THÔNG ADT & Đặng Huy Thắng & 100905796 & Sản xuất khác \\
\hline 9 & 9 & CÔNG TY CP KÉT NÓI NHÂN TÀI & Tiêu Yến Trinh & 305202145 & Kinh doanh tổng hợp khác \\
\hline 10 & 10 & CÔNG TY TNHH THIÉT LÂP & Nguyễn Hoàng Ẩn & 1500441413 & $\begin{array}{l}\text { Sản xuất, kinh doanh, chế biến gạo, bột mỳ, } \\
\text { ngũ cốc... }\end{array}$ \\
\hline 11 & 11 & $\begin{array}{l}\text { CÔNG TY TNHH-CHÉ BIÉN THỦY SẢN VÀ XNK } \\
\text { TRANG KHANH }\end{array}$ & Trần Tuấn Khanh & 1900287501 & $\begin{array}{l}\text { Nuôi trồng, chế biến, kinh doanh thuỷ sản và } \\
\text { các sản phẩm thit }\end{array}$ \\
\hline 12 & 12 & $\begin{array}{l}\text { CÔNG TY SẢN XUÂT THƯƠNG MAII VÀ ĐÀU TƯ } \\
\text { TAM MINH }\end{array}$ & Lưu Tuấn Anh & 4300327589 & Sản xuất đồ gỗ, nôi thất \\
\hline 13 & 13 & $\begin{array}{l}\text { CÔNG TY TNHH THƯO'NG MAI SẢN XUÂT HÙNG } \\
\text { HUY }\end{array}$ & Voòng $\mathrm{A} \mathrm{Hoa}$ & 302259111 & Sản xuất và gia công giầy, dép \\
\hline
\end{tabular}


Citation: Huy DTN (2014) Changing Tax Rates Impacts on the Risk Level of Vietnam Construction Material Firms. J Entrepren Organiz Manag 3: 110. doi: 10.4172/2169-026X.1000110

Page 4 of 9

\begin{tabular}{|c|c|c|c|c|c|}
\hline 14 & 14 & CÔNG TY TNHH THƯƠNG MAII HOÀNG ĐAO & Hồ Hoàng Bách & 301434963 & $\begin{array}{l}\text { Sản xuất, kinh doanh thuốc lá, nguyên liêu } \\
\text { thuốc lá }\end{array}$ \\
\hline 15 & 15 & $\begin{array}{l}\text { CÔNG TY TNHH THƯƠNG MAI XÂY DỰNG DIẸU } \\
\text { LONG }\end{array}$ & Đặng Thị Linh Phương & 302000905 & Kinh doanh tổng hơp khác \\
\hline 16 & 16 & $\begin{array}{l}\text { CÔNG TY CP XÂY DƯNG NHÀ VÀ PHÁT TRIẺN } \\
\text { HA TẦNG HÀ NÔI }\end{array}$ & Phạm Thanh Nam & 101370536 & $\begin{array}{l}\text { Xây dưng nhà và các công trình kỹ thuât dân } \\
\text { dung: hoat đông xây dưng chuyên dung }\end{array}$ \\
\hline 17 & 17 & $\begin{array}{l}\text { CÔNG TY CP XĂNG DÀU THƯƠNG MẠI SÔNG } \\
\text { TRÀ }\end{array}$ & Nguyễn Anh & 4300334642 & $\begin{array}{l}\text { Kinh doanh xăng dầu, khí đốt, nhiên liệu và } \\
\text { các sản phẩm liên quan }\end{array}$ \\
\hline 18 & 18 & CÔNG TY CP SO'I PHÚ THANH & Nguyễn Văn Cường & 3300471069 & Sản xuất sợi, dẹt \\
\hline 19 & 19 & $\begin{array}{l}\text { CÔNG TY TNHH DU LI!CH VÀ THƯO'NG MAI SÔNG } \\
\text { HÖNG }\end{array}$ & Lê Công Hoàng & 100237411 & $\begin{array}{l}\text { Kinh doanh xăng dầu, khí đốt, nhiên liệu và } \\
\text { các sản phẩm liên quan }\end{array}$ \\
\hline 20 & 20 & CÔNG TY CP DÀUU THƯ'C VÂT BİNH ĐINH & Nguyễn Thị Thùy Giao & 4100399995 & $\begin{array}{l}\text { Sản xuất thưc phẩm chế biến: sữa, đường, cà } \\
\text { phê, dầu ăn, bánh keo... }\end{array}$ \\
\hline 21 & 21 & CÔNG TY CP VĀT TƯ MỎ ĐIIA CHÂT & Lê Nhật Tân & 101453768 & Kinh doanh tổng hợp khác \\
\hline 22 & 22 & CÔNG TY TNHH MTV SÀI GÒN BÉN TRE & Nguyễn Ngọc Minh Hùng & 1300419650 & Bán lẻ, kinh doanh siêu thi \\
\hline 23 & 23 & CÔNG TY TNHH HẢI VIET & Nguyễn Văn Rỉ & 3700443193 & Kinh doanh nông, lâm sản \\
\hline 24 & 24 & CÔNG TY TNHH MAY PHÙ ĐỎNG & Dương Đức Thanh & 100598947 & Ngành may \\
\hline 25 & 25 & $\begin{array}{l}\text { CÔNG TY TNHH THƯO'NG MAI VÀ DICH VU KY } \\
\text { THUÂT TESCO }\end{array}$ & Trần Thanh Bình & 101507773 & Sản xuất, kinh doanh ôtô, phu tùng ôtô \\
\hline 26 & 26 & CÔNG TY TNHH THƯƠNG MAI PHÚC THÁl & Bùi Xuân Huy & 600346391 & $\begin{array}{l}\text { Kinh doanh xăng dầu, khí đốt, nhiên liêu và } \\
\text { các sản phẩm liên quan }\end{array}$ \\
\hline 27 & 27 & CÔNG TY TNHH MINH ĐĂNG & Nguyễn Thị Cẩm Nhung & 2200258144 & $\begin{array}{l}\text { Nuôi trồng, chế biến, kinh doanh thuỷ sản và } \\
\text { các sản phẩm thit }\end{array}$ \\
\hline 28 & 28 & $\begin{array}{l}\text { CÔNG TY TNHH THƯƠNG MAI VÀ DICH VU VÂN } \\
\text { TẢI NGOC KHÁNH }\end{array}$ & Trần Ngọc Huân & 4000377194 & $\begin{array}{l}\text { Kinh doanh xăng dầu, khí đốt, nhiên liêu và } \\
\text { các sản phẩm liên quan }\end{array}$ \\
\hline 29 & 29 & $\begin{array}{l}\text { CÔNG TY TNHH THƯO'NG MAI DICH VU VÂN } \\
\text { PHÁT }\end{array}$ & Cao Ngọc Anh & 303332212 & Kinh doanh tổng hơp khác \\
\hline 30 & 30 & $\begin{array}{l}\text { CÔNG TY CP VÂN TẢI VÀ DICH VU PETROLIMEX } \\
\text { NGHE TĨNH }\end{array}$ & Võ Văn Tân & 2900428497 & $\begin{array}{l}\text { Kinh doanh xăng dầu, khí đốt, nhiên liêu và } \\
\text { các sản phẩm liên quan }\end{array}$ \\
\hline 31 & 31 & $\begin{array}{l}\text { CÔNG TY TNHH THƯO'NG MAI SẢN XUÁT TÂN } \\
\text { BİNH }\end{array}$ & Trần Trí Thức & 3900326063 & Sản xuất đồ gỗ, nôi thất \\
\hline 32 & 32 & CÔNG TY TNHH NGUYÊN LIÊU GIÂY DUNG QUÂT & Nguyễn Nị & 4300308498 & Sản xuất đồ gỗ, nôi thất \\
\hline 33 & 33 & $\begin{array}{l}\text { CÔNG TY TNHH BENH VIEEN ĐA KHOA TƯ NHÂN } \\
\text { AN SINH }\end{array}$ & Ngô Đăng Sơn Anh & 302774433 & Sản xuất khác \\
\hline 34 & 34 & CÔNG TY TNHH IN LUA NHÂT PHƯƠNG & Huỳnh Thị Thanh Thuỷ & 301441840 & Sản xuất giấy, in ấn, xuất bản \\
\hline 35 & 35 & CÔNG TY CP DÀU KHÍ SÀI GÒN-PHÚ YÊN & Trần Công Chưởng & 4400344901 & $\begin{array}{l}\text { Kinh doanh xăng dầu, khí đốt, nhiên liêu và } \\
\text { các sản phẩm liên quan }\end{array}$ \\
\hline 36 & 36 & CÔNG TY CP BÊ TÔNG ĐĂNG HẢl & Phan Văn Khanh & 400619838 & $\begin{array}{l}\text { Sản xuất, kinh doanh vât liêu xây dưng: xi } \\
\text { măng, gach xây, đá, cát... }\end{array}$ \\
\hline 37 & 37 & CÔNG TY CP GIÀY BİNH ĐINH & Võ Ngọc Thủy & 4100258761 & Sản xuất và gia công giầy, dép \\
\hline 38 & 38 & CÔNG TY CP ĐÀUU TƯ PHÁT TRIÉN THIÊN SƠN & Vũ Văn Tuyên & 101536301 & $\begin{array}{l}\text { Sản xuất, kinh doanh vât liêu xây dưng: xi } \\
\text { măng, gach xây, đá, cát... }\end{array}$ \\
\hline 39 & 39 & HTX THƯƠNG MAI ĐÔ THÀNH & Hàng Thanh Dân & 302804374 & $\begin{array}{l}\text { Kinh doanh thưc phẩm chế biến: sữa, đường, } \\
\text { cà phê, dầu ăn, bánh keo...... }\end{array}$ \\
\hline 40 & 40 & CÔNG TY CP XÂY DƯNG TỎNG HƠP 269 & Trương Duy Niệm & 3000244523 & $\begin{array}{l}\text { Xây dưng nhà và các công trình kỹ thuât dân } \\
\text { dung; hoat đông xây dưng chuyên dung }\end{array}$ \\
\hline
\end{tabular}

Table 4: Top 40 among top 500 SMEs with the highest growth speed in 2012 (source: Vietnamnet and Viet Nam report).

VN construction material market as following: (Table 8). The exhibit shows us that comparing to equity beta, asset beta values of firms had a big decrease.

- Scenario 2: tax rate increases up to $28 \%$

If corporate tax rates increases up to $28 \%$, all beta values of total 57 listed firms on VN construction material market as below: (Table 9).

- Scenario 3: tax rate decreases down to $20 \%$

If corporate tax rate decreases down to $20 \%$, all beta values of total 57 listed firms on the construction material market in $\mathrm{VN}$ as following: (Table 10).

All three above tables and data show that values of equity and asset beta in the case of increasing tax rate up to $28 \%$ or decreasing rate down to $20 \%$ have small fluctuation.

\section{Comparing Statistical Results in 3 Scenarios of Changing Tax Rate}

Based on above results, we find out:

Equity beta mean values in all 3 scenarios are acceptable despite of in the case tax rate of $25 \%$, beta is higher than ( $>$ ) 1 , but asset beta mean values are much smaller. In the case of current tax rate of $25 \%$, equity beta value fluctuates in a wide range from 0,129 ( $\mathrm{min}$ ) up to 3,693 ( $\max$ value is much higher) and asset beta fluctuates from $0,041(\mathrm{~min})$ up to $1,807(\max )$. If corporate tax rate increases to $28 \%$, equity beta moves from $0,132(\min )$ up to $3,693(\max )$ and asset beta moves from $0,030(\min )$ up to 1,807 ( $\max$ ). Hence, we note that there is a little increasing change in equity beta min value if corporate tax increases. When tax rate decreases down to $20 \%$, equity beta value changes from $0,124(\min )$ up to $3,693(\max )$ and asset beta changes from $0,028(\mathrm{~min})$ up to $1,807(\max )$. So, there are 
Citation: Huy DTN (2014) Changing Tax Rates Impacts on the Risk Level of Vietnam Construction Material Firms. J Entrepren Organiz Manag 3: 110. doi: 10.4172/2169-026X.1000110

Page 5 of 9

\begin{tabular}{|c|c|c|c|c|c|}
\hline Order No. & Company stock code & Equity beta & Asset beta (assume debt beta $=0$ ) & Note & Financial leverage \\
\hline 1 & DIC & 0,986 & 0,337 & & $65,8 \%$ \\
\hline 2 & LBM & 1,186 & 0,783 & & $34,0 \%$ \\
\hline 3 & NAV & 0,895 & 0,539 & & $39,8 \%$ \\
\hline 4 & DXV & 1,135 & 0,185 & & $83,7 \%$ \\
\hline 5 & HT1 & 0,599 & 0,087 & & $85,4 \%$ \\
\hline 6 & CVT & 2,504 & 1,031 & & $58,8 \%$ \\
\hline 7 & DC4 & 1,007 & 0,345 & & $65,7 \%$ \\
\hline 8 & HPS & 0,815 & 0,697 & & $14,5 \%$ \\
\hline 9 & KBT & 1,019 & 0,639 & VE1 as comparable & $37,4 \%$ \\
\hline 10 & PPG & 0,755 & 0,354 & & $53,1 \%$ \\
\hline 11 & SDN & 0,533 & 0,281 & & $47,4 \%$ \\
\hline 12 & SKS & 0,761 & 0,358 & & $53,0 \%$ \\
\hline 13 & VXB & 0,355 & 0,141 & SKS as comparable & $60,4 \%$ \\
\hline 14 & DHA & 0,837 & 0,704 & & $15,8 \%$ \\
\hline 15 & CTI & 0,129 & 0,041 & LM3 as comparable & $68,2 \%$ \\
\hline 16 & DCT & 0,869 & 0,316 & & $63,7 \%$ \\
\hline 17 & SCL & 1,007 & 0,550 & DC4 as comparable & $45,4 \%$ \\
\hline 18 & HVX & 0,816 & 0,567 & DTC as comparable & $30,5 \%$ \\
\hline 19 & NHC & 0,717 & 0,549 & & $23,4 \%$ \\
\hline 20 & BHV & 1,308 & 0,412 & & $68,5 \%$ \\
\hline 21 & XMC & 1,095 & 0,211 & & $80,8 \%$ \\
\hline 22 & ACC & 0,816 & 0,602 & $\mathrm{HVX}$ as comparable & $26,3 \%$ \\
\hline 23 & BBS & 0,689 & 0,358 & & $48,0 \%$ \\
\hline 24 & $\mathrm{BCC}$ & 0,851 & 0,148 & & $82,6 \%$ \\
\hline 25 & BHC & 0,677 & 0,153 & & $77,3 \%$ \\
\hline 26 & BHT & 0,816 & 0,137 & DTC as comparable & $83,2 \%$ \\
\hline 27 & BT6 & 0,407 & 0,126 & & $68,9 \%$ \\
\hline 28 & BTS & 0,880 & 0,188 & & $78,6 \%$ \\
\hline 29 & $\mathrm{CCM}$ & 1,095 & 0,554 & & $49,5 \%$ \\
\hline 30 & CYC & 0,788 & 0,239 & & $69,6 \%$ \\
\hline 31 & DAC & 1,027 & 0,559 & & $45,6 \%$ \\
\hline 32 & DTC & 0,816 & 0,161 & & $80,3 \%$ \\
\hline 33 & GMX & 1,427 & 0,885 & SDY as comparable & $38,0 \%$ \\
\hline 34 & $\mathrm{HCC}$ & 1,022 & 0,534 & & $47,7 \%$ \\
\hline 35 & HHL & 1,787 & 0,692 & & $61,3 \%$ \\
\hline 36 & HLY & 0,948 & 0,446 & & $52,9 \%$ \\
\hline 37 & HOM & 0,585 & 0,243 & & $58,5 \%$ \\
\hline 38 & MCC & 1,308 & 1,181 & $\mathrm{BHV}$ as comparable & $9,7 \%$ \\
\hline 39 & MCL & 0,717 & 0,378 & $\mathrm{NHC}$ as comparable & $47,3 \%$ \\
\hline 40 & NNC & 0,816 & 0,619 & DTC as comparable & $24,1 \%$ \\
\hline 41 & QNC & 0,939 & 0,105 & & $88,8 \%$ \\
\hline 42 & SCC & 0,943 & 0,710 & & $24,7 \%$ \\
\hline 43 & SCJ & 1,390 & 0,703 & & $49,4 \%$ \\
\hline 44 & SDY & 1,427 & 0,479 & & $66,4 \%$ \\
\hline 45 & SHN & 3,693 & 1,807 & & $51,1 \%$ \\
\hline 46 & TBX & 0,493 & 0,248 & & $49,6 \%$ \\
\hline 47 & TCR & 0,759 & 0,376 & & $50,4 \%$ \\
\hline 48 & TLT & 1,448 & 0,088 & & $93,9 \%$ \\
\hline 49 & TMX & 1,559 & 0,568 & & $63,6 \%$ \\
\hline 50 & TSM & 1,787 & 1,333 & $\mathrm{HHL}$ as comparable & $25,4 \%$ \\
\hline 51 & TTC & 0,708 & 0,241 & & $66,0 \%$ \\
\hline 52 & TXM & 1,013 & 0,377 & & $62,8 \%$ \\
\hline 53 & VCS & 1,177 & 0,500 & & $57,6 \%$ \\
\hline 54 & VHL & 0,538 & 0,137 & & $74,5 \%$ \\
\hline 55 & VIT & 0,541 & 0,126 & & $76,8 \%$ \\
\hline 56 & VTS & 1,078 & 0,647 & & $40,0 \%$ \\
\hline 57 & YBC & 1,310 & 0,227 & & $82,7 \%$ \\
\hline
\end{tabular}

Table 5: Risk and financial leverage of 57 listed construction material firms on VN stock exchange period 2007-2011. 
Citation: Huy DTN (2014) Changing Tax Rates Impacts on the Risk Level of Vietnam Construction Material Firms. J Entrepren Organiz Manag 3: 110. doi: 10.4172/2169-026X.1000110

Page 6 of 9

\begin{tabular}{|c|c|c|c|c|c|c|c|}
\hline \multirow[b]{2}{*}{ Order No. } & \multirow{2}{*}{$\begin{array}{c}\text { Company stock } \\
\text { code }\end{array}$} & \multicolumn{2}{|c|}{$\mathbf{t}=\mathbf{2 5 \%}$} & \multicolumn{2}{|c|}{$t=28 \%$} & \multicolumn{2}{|c|}{$t=20 \%$} \\
\hline & & Equity beta & Asset beta & $\begin{array}{c}\text { Increase/Decrease } \\
\text { (equity beta) }\end{array}$ & $\begin{array}{l}\text { Increase/Decrease } \\
\text { (asset beta) }\end{array}$ & $\begin{array}{c}\text { Increase/Decrease } \\
\text { (equity beta) }\end{array}$ & $\begin{array}{c}\text { Increase/Decrease } \\
\text { (asset beta) }\end{array}$ \\
\hline 1 & DIC & 0,9856 & 0,3373 & 0,0000 & 0,0000 & 0,0000 & 0,0000 \\
\hline 2 & LBM & 1,1862 & 0,7832 & 0,0000 & 0,0000 & 0,0000 & 0,0000 \\
\hline 3 & NAV & 0,8954 & 0,5393 & 0,0000 & 0,0000 & 0,0000 & 0,0000 \\
\hline 4 & DXV & 1,1349 & 0,1847 & 0,0000 & 0,0000 & 0,0000 & 0,0000 \\
\hline 5 & HT1 & 0,5991 & 0,0872 & 0,0000 & 0,0000 & 0,0000 & 0,0000 \\
\hline 6 & CVT & 2,5036 & 1,0314 & 0,0000 & 0,0000 & 0,0000 & 0,0000 \\
\hline 7 & DC4 & 1,0068 & 0,3450 & 0,0000 & 0,0000 & 0,0000 & 0,0000 \\
\hline 8 & HPS & 0,8155 & 0,6968 & 0,0000 & 0,0000 & 0,0000 & 0,0000 \\
\hline 9 & KBT & 1,0193 & 0,6385 & 0,0128 & 0,0080 & $-0,0206$ & $-0,0129$ \\
\hline 10 & PPG & 0,7551 & 0,3543 & 0,0000 & 0,0000 & 0,0000 & 0,0000 \\
\hline 11 & SDN & 0,5330 & 0,2806 & 0,0000 & 0,0000 & 0,0000 & 0,0000 \\
\hline 12 & SKS & 0,7611 & 0,3576 & 0,0000 & 0,0000 & 0,0000 & 0,0000 \\
\hline 13 & VXB & 0,3553 & 0,1408 & 0,0077 & 0,0031 & $-0,0122$ & $-0,0048$ \\
\hline 14 & DHA & 0,8366 & 0,7045 & 0,0000 & 0,0000 & 0,0000 & 0,0000 \\
\hline 15 & CTI & 0,1289 & 0,0410 & 0,0033 & 0,0010 & $-0,0051$ & $-0,0016$ \\
\hline 16 & DCT & 0,8690 & 0,3157 & 0,0000 & 0,0003 & 0,0000 & 0,0000 \\
\hline 17 & $\mathrm{SCL}$ & 1,0068 & 0,5496 & $-0,3771$ & $-0,2059$ & $-0,4023$ & $-0,2196$ \\
\hline 18 & $H V X$ & 0,8158 & 0,5666 & $-0,1962$ & $-0,1363$ & $-0,2123$ & $-0,1475$ \\
\hline 19 & $\mathrm{NHC}$ & 0,7168 & 0,5492 & 0,0000 & 0,0000 & 0,0000 & 0,0000 \\
\hline 20 & $\mathrm{BHV}$ & 1,3078 & 0,4118 & 0,0000 & 0,0000 & 0,0000 & 0,0000 \\
\hline 21 & XMC & 1,0947 & 0,2106 & 0,0000 & 0,0000 & 0,0000 & 0,0000 \\
\hline 22 & ACC & 0,8158 & 0,6015 & $-0,3226$ & $-0,2379$ & $-0,3461$ & $-0,2552$ \\
\hline 23 & BBS & 0,6892 & 0,3584 & 0,0000 & 0,0000 & 0,0000 & 0,0000 \\
\hline 24 & BCC & 0,8510 & 0,1478 & 0,0000 & 0,0000 & 0,0000 & 0,0000 \\
\hline 25 & $\mathrm{BHC}$ & 0,6769 & 0,1534 & 0,0000 & 0,0000 & 0,0000 & 0,0000 \\
\hline 26 & BHT & 0,8158 & 0,1371 & $-0,6370$ & $-0,1071$ & $-0,6513$ & $-0,1095$ \\
\hline 27 & BT6 & 0,4072 & 0,1265 & 0,0000 & 0,0000 & 0,0000 & 0,0000 \\
\hline 28 & BTS & 0,8804 & 0,1882 & 0,0000 & 0,0000 & 0,0000 & 0,0000 \\
\hline 29 & $\mathrm{CCM}$ & 1,0955 & 0,5536 & 0,0000 & 0,0000 & 0,0000 & 0,0000 \\
\hline 30 & CYC & 0,7880 & 0,2392 & 0,0000 & 0,0000 & 0,0000 & 0,0000 \\
\hline 31 & DAC & 1,0273 & 0,5592 & 0,0000 & 0,0000 & 0,0000 & 0,0000 \\
\hline 32 & DTC & 0,8158 & 0,1609 & 0,0000 & 0,0000 & 0,0000 & 0,0000 \\
\hline 33 & GMX & 1,4265 & 0,8848 & $-0,4365$ & $-0,2707$ & $-0,4690$ & $-0,2909$ \\
\hline 34 & $\mathrm{HCC}$ & 1,0215 & 0,5342 & 0,0000 & 0,0000 & 0,0000 & 0,0000 \\
\hline 35 & $\mathrm{HHL}$ & 1,7873 & 0,6920 & 0,0000 & 0,0000 & 0,0000 & 0,0000 \\
\hline 36 & HLY & 0,9477 & 0,4461 & 0,0000 & 0,0000 & 0,0000 & 0,0000 \\
\hline 37 & HOM & 0,5853 & 0,2430 & 0,0000 & 0,0000 & 0,0000 & 0,0000 \\
\hline 38 & MCC & 1,3078 & 1,1813 & $-0,0936$ & $-0,0846$ & $-0,1032$ & $-0,0932$ \\
\hline 39 & $\mathrm{MCL}$ & 0,7168 & 0,3776 & $-0,2815$ & $-0,1483$ & $-0,2997$ & $-0,1579$ \\
\hline 40 & NNC & 0,8158 & 0,6192 & $-0,1518$ & $-0,1152$ & $-0,1653$ & $-0,1254$ \\
\hline 41 & QNC & 0,9395 & 0,1051 & 0,0000 & 0,0000 & 0,0000 & 0,0000 \\
\hline 42 & SCC & 0,9429 & 0,7098 & 0,0000 & 0,0000 & 0,0000 & 0,0000 \\
\hline 43 & SCJ & 1,3904 & 0,7031 & 0,0000 & 0,0000 & 0,0000 & 0,0000 \\
\hline 44 & SDY & 1,4265 & 0,4789 & 0,0000 & 0,0000 & 0,0000 & 0,0000 \\
\hline 45 & SHN & 3,6926 & 1,8072 & 0,0000 & 0,0000 & 0,0000 & 0,0000 \\
\hline 46 & TBX & 0,4932 & 0,2484 & 0,0000 & 0,0000 & 0,0000 & 0,0000 \\
\hline 47 & TCR & 0,7593 & 0,3763 & 0,0000 & 0,0000 & 0,0000 & 0,0000 \\
\hline 48 & TLT & 1,4483 & 0,0880 & 0,0000 & 0,0000 & 0,0000 & 0,0000 \\
\hline 49 & TMX & 1,5594 & 0,5682 & 0,0000 & 0,0000 & 0,0000 & 0,0000 \\
\hline 50 & TSM & 1,7873 & 1,3326 & $-0,3525$ & $-0,2628$ & $-0,3832$ & $-0,2857$ \\
\hline 51 & TTC & 0,7075 & 0,2409 & 0,0000 & 0,0000 & 0,0000 & 0,0000 \\
\hline 52 & TXM & 1,0135 & 0,3774 & 0,0000 & 0,0000 & 0,0000 & 0,0000 \\
\hline 53 & VCS & 1,1768 & 0,4995 & 0,0000 & 0,0000 & 0,0000 & 0,0000 \\
\hline 54 & VHL & 0,5380 & 0,1371 & 0,0000 & 0,0000 & 0,0000 & 0,0000 \\
\hline 55 & VIT & 0,5415 & 0,1258 & 0,0000 & 0,0000 & 0,0000 & 0,0000 \\
\hline 56 & VTS & 1,0780 & 0,6468 & 0,0000 & 0,0000 & 0,0000 & 0,0000 \\
\hline \multirow[t]{2}{*}{57} & YBC & 1,3097 & 0,2270 & 0,0000 & 0,0000 & 0,0000 & 0,0000 \\
\hline & \multicolumn{3}{|c|}{ Average } & $-0,0496$ & $-0,0273$ & $-0,0539$ & $-0,0299$ \\
\hline
\end{tabular}

Table 6: Increase/decrease risk level of listed construction material firms under changing scenarios of tax rates : 25\%, 28\%, 20\% period $2007-2011$. 
Page 7 of 9

\begin{tabular}{|c|c|c|c|}
\hline Statistic results & Equity beta & $\begin{array}{c}\text { Asset beta } \\
\text { (assume debt beta } \mathbf{0} \text { ) }\end{array}$ & Difference \\
\hline MAX & 1,770 & 1,575 & 0,195 \\
\hline MIN & 0,062 & 0,016 & 0,046 \\
\hline MEAN & 0,750 & 0,362 & 0,388 \\
\hline VAR & 0,2624 & 0,1151 & 0,147 \\
\hline \multicolumn{3}{|c|}{ Note: Sample size : 45} \\
\hline
\end{tabular}

Table 7: Statistical results (tax rate $=20 \%$ ) in the real estate industry 2007-2009.

\begin{tabular}{|c|c|c|c|}
\hline Order No. & Company stock code & Equity beta & $\begin{array}{c}\text { Asset beta } \\
\text { (assume debt beta }=0 \text { ) }\end{array}$ \\
\hline 1 & DIC & 0,986 & 0,337 \\
\hline 2 & LBM & 1,186 & 0,783 \\
\hline 3 & NAV & 0,895 & 0,539 \\
\hline 4 & DXV & 1,135 & 0,185 \\
\hline 5 & HT1 & 0,599 & 0,087 \\
\hline 6 & CVT & 2,504 & 1,031 \\
\hline 7 & DC4 & 1,007 & 0,345 \\
\hline 8 & HPS & 0,815 & 0,697 \\
\hline 9 & KBT & 1,019 & 0,639 \\
\hline 10 & PPG & 0,755 & 0,354 \\
\hline 11 & SDN & 0,533 & 0,281 \\
\hline 12 & SKS & 0,761 & 0,358 \\
\hline 13 & VXB & 0,355 & 0,141 \\
\hline 14 & DHA & 0,837 & 0,704 \\
\hline 15 & CTI & 0,129 & 0,041 \\
\hline 16 & DCT & 0,869 & 0,316 \\
\hline 17 & SCL & 1,007 & 0,550 \\
\hline 18 & HVX & 0,816 & 0,567 \\
\hline 19 & NHC & 0,717 & 0,549 \\
\hline 20 & BHV & 1,308 & 0,412 \\
\hline 21 & XMC & 1,095 & 0,211 \\
\hline 22 & ACC & 0,816 & 0,602 \\
\hline 23 & BBS & 0,689 & 0,358 \\
\hline 24 & BCC & 0,851 & 0,148 \\
\hline 25 & BHC & 0,677 & 0,153 \\
\hline 26 & BHT & 0,816 & 0,137 \\
\hline 27 & BT6 & 0,407 & 0,126 \\
\hline 28 & BTS & 0,880 & 0,188 \\
\hline 29 & CCM & 1,095 & 0,554 \\
\hline 30 & CYC & 0,788 & 0,239 \\
\hline 31 & DAC & 1,027 & 0,559 \\
\hline 32 & DTC & 0,816 & 0,161 \\
\hline 33 & GMX & 1,427 & 0,885 \\
\hline 34 & HCC & 1,022 & 0,534 \\
\hline 35 & HHL & 1,787 & 0,692 \\
\hline 36 & HLY & 0,948 & 0,446 \\
\hline 37 & HOM & 0,585 & 0,243 \\
\hline 38 & MCC & 1,308 & 1,181 \\
\hline 39 & MCL & 0,717 & 0,378 \\
\hline 40 & NNC & 0,816 & 0,619 \\
\hline 41 & QNC & 0,939 & 0,105 \\
\hline 42 & SCC & 0,943 & 0,710 \\
\hline 43 & SCJ & 1,390 & 0,703 \\
\hline 44 & SDY & 1,427 & 0,479 \\
\hline 45 & SHN & 3,693 & 1,807 \\
\hline 46 & TBX & 0,493 & 0,248 \\
\hline 47 & TCR & 0,759 & 0,376 \\
\hline
\end{tabular}

\begin{tabular}{|r|l|r|r|}
\hline 48 & TLT & 1,448 & 0,088 \\
\hline 49 & TMX & 1,559 & 0,568 \\
\hline 50 & TSM & 1,787 & 1,333 \\
\hline 51 & TTC & 0,708 & 0,241 \\
\hline 52 & TXM & 1,013 & 0,377 \\
\hline 53 & VCS & 1,177 & 0,500 \\
\hline 54 & VHL & 0,538 & 0,137 \\
\hline 55 & VIT & 0,541 & 0,126 \\
\hline 56 & VTS & 1,078 & 0,647 \\
\hline 57 & YBC & 1,310 & 0,227 \\
\hline
\end{tabular}

Table 8: Market risk of listed companies on $\mathrm{VN}$ construction material market $(\mathrm{t}=$ $25 \%)$.

small decreasing changes in equity/asset beta min values when tax decreases in scenario 3 (Table 11).

Beside, Exhibit 7 informs us that in the case $28 \%$ tax rate, average equity beta value of 57 listed firms decreases up to 0,05 while average asset beta value of these 57 firms increase slightly up to 0,027 . Then, when tax rate reduces to $20 \%$, average equity beta value of 57 listed firms reduce to 0,05 and average asset beta value of 57 firms down to 0,03 (Table 12).

The below chart 1 shows us : when tax rate decreases down to $20 \%$, average equity and asset beta values decrease slightly $(0,957$ and 0,426$)$ compared to those at the initial rate of $25 \%(1,011$ and 0,456$)$. At the same time, when tax rate increases up to $28 \%$, average equity and asset beta decrease just slightly (to 0,961 and 0,429 ). However, the fluctuation of equity beta value $(0,298)$ in the case of $20 \%$ tax rate is higher than $(>)$ the results in the rest 2 tax rate cases (Table 13 and Figure 1,4).

\section{Risk Analysis}

In the case of decreasing tax rate, (20\%), the market and companies can receive more benefits such as generating more jobs and compensation, but the government budget can have deficit and the government has to cut expenses. Changes in tax rates can have both positive and negative impacts on the local market.

In the case of increasing tax rate (28\%), the government will have budget to finance public expenditures but the tax could reduce both demand and supply.

The debt level of business sector also needed to be taken into account if the government would like to change the tax rates.

\section{Conclusion and Policy Suggestion}

In summary, the government continues to increase the effectiveness of building the legal system and regulation and macro policies supporting the plan of developing both the construction and the construction material market. The Ministry of Finance Continue to increase the effectiveness of fiscal policies and tax policies which are needed to combine with other macro policies at the same time, although we could note that in this study when tax rate is going to increase up to $28 \%$, the value of equity beta mean decreases down to 0,961 , from 1,011 and asset beta mean decreases down to 0,429 , from 0,456 . Furthermore, it has to pay attention to the degree of using leverage (operation and financial leverage) in the industry before it decides to change the tax rates. Certainly, the government bodies could choose either changing tax rates or cutting public expenditures as changing tax rates might have both negative and positive impacts.

The State Bank of Viet Nam continues to increase the effectiveness 
Citation: Huy DTN (2014) Changing Tax Rates Impacts on the Risk Level of Vietnam Construction Material Firms. J Entrepren Organiz Manag 3: 110. doi: 10.4172/2169-026X.1000110

Page 8 of 9

\begin{tabular}{|c|c|c|c|}
\hline Order No. & Company stock code & Equity beta & $\begin{array}{c}\text { Asset beta } \\
(\text { assume debt beta }=0)\end{array}$ \\
\hline 1 & DIC & 0,986 & 0,337 \\
\hline 2 & LBM & 1,186 & 0,783 \\
\hline 3 & NAV & 0,895 & 0,539 \\
\hline 4 & DXV & 1,135 & 0,185 \\
\hline 5 & HT1 & 0,599 & 0,087 \\
\hline 6 & CVT & 2,504 & 1,031 \\
\hline 7 & DC4 & 1,007 & 0,345 \\
\hline 8 & HPS & 0,815 & 0,697 \\
\hline 9 & KBT & 1,032 & 0,646 \\
\hline 10 & PPG & 0,755 & 0,354 \\
\hline 11 & SDN & 0,533 & 0,281 \\
\hline 12 & SKS & 0,761 & 0,358 \\
\hline 13 & VXB & 0,363 & 0,144 \\
\hline 14 & DHA & 0,837 & 0,704 \\
\hline 15 & CTI & 0,132 & 0,042 \\
\hline 16 & DCT & 0,869 & 0,316 \\
\hline 17 & SCL & 0,630 & 0,344 \\
\hline 18 & HVX & 0,620 & 0,430 \\
\hline 19 & NHC & 0,717 & 0,549 \\
\hline 20 & BHV & 1,308 & 0,412 \\
\hline 21 & XMC & 1,095 & 0,211 \\
\hline 22 & ACC & 0,493 & 0,364 \\
\hline 23 & BBS & 0,689 & 0,358 \\
\hline 24 & BCC & 0,851 & 0,148 \\
\hline 25 & BHC & 0,677 & 0,153 \\
\hline 26 & BHT & 0,179 & 0,030 \\
\hline 27 & BT6 & 0,407 & 0,126 \\
\hline 28 & BTS & 0,880 & 0,188 \\
\hline 29 & CCM & 1,095 & 0,554 \\
\hline 30 & CYC & 0,788 & 0,239 \\
\hline 31 & DAC & 1,027 & 0,559 \\
\hline 32 & DTC & 0,816 & 0,161 \\
\hline 33 & GMX & 0,990 & 0,614 \\
\hline 34 & HCC & 1,022 & 0,534 \\
\hline 35 & HHL & 1,787 & 0,692 \\
\hline 36 & HLY & 0,948 & 0,446 \\
\hline 37 & HOM & 0,585 & 0,243 \\
\hline 38 & MCC & 1,214 & 1,097 \\
\hline 39 & MCL & 0,435 & 0,229 \\
\hline 40 & NNC & 0,664 & 0,504 \\
\hline 41 & QNC & 0,939 & 0,105 \\
\hline 42 & SCC & 0,943 & 0,710 \\
\hline 43 & SCJ & 1,390 & 0,703 \\
\hline 44 & SDY & 1,427 & 0,479 \\
\hline 45 & SHN & 3,693 & 1,807 \\
\hline 46 & TBX & 0,493 & 0,248 \\
\hline 47 & TCR & 0,759 & 0,376 \\
\hline 48 & TLT & 1,448 & 0,088 \\
\hline 49 & TMX & 1,559 & 0,568 \\
\hline 50 & TSM & 1,435 & 1,070 \\
\hline 51 & TTC & 0,708 & 0,241 \\
\hline 52 & TXM & 1,013 & 0,377 \\
\hline 53 & vcs & 1,177 & 0,500 \\
\hline 54 & VHL & 0,538 & 0,137 \\
\hline 55 & VIT & 0,541 & 0,126 \\
\hline 56 & VTS & 1,078 & 0,647 \\
\hline 57 & YBC & 1,310 & 0,227 \\
\hline
\end{tabular}

Table 9: Market risks of listed construction material firms $(t=28 \%)$.

\begin{tabular}{|c|c|c|c|}
\hline Order No. & Company stock code & Equity beta & $\begin{array}{c}\text { Asset beta } \\
\text { (assume debt beta }=0 \text { ) }\end{array}$ \\
\hline 1 & DIC & 0,986 & 0,337 \\
\hline 2 & LBM & 1,186 & 0,783 \\
\hline 3 & NAV & 0,895 & 0,539 \\
\hline 4 & DXV & 1,135 & 0,185 \\
\hline 5 & HT1 & 0,599 & 0,087 \\
\hline 6 & CVT & 2,504 & 1,031 \\
\hline 7 & DC4 & 1,007 & 0,345 \\
\hline 8 & HPS & 0,815 & 0,697 \\
\hline 9 & KBT & 0,999 & 0,626 \\
\hline 10 & PPG & 0,755 & 0,354 \\
\hline 11 & SDN & 0,533 & 0,281 \\
\hline 12 & SKS & 0,761 & 0,358 \\
\hline 13 & VXB & 0,343 & 0,136 \\
\hline 14 & DHA & 0,837 & 0,704 \\
\hline 15 & CTI & 0,124 & 0,039 \\
\hline 16 & DCT & 0,869 & 0,316 \\
\hline 17 & SCL & 0,604 & 0,330 \\
\hline 18 & HVX & 0,603 & 0,419 \\
\hline 19 & NHC & 0,717 & 0,549 \\
\hline 20 & BHV & 1,308 & 0,412 \\
\hline 21 & XMC & 1,095 & 0,211 \\
\hline 22 & ACC & 0,470 & 0,346 \\
\hline 23 & BBS & 0,689 & 0,358 \\
\hline 24 & BCC & 0,851 & 0,148 \\
\hline 25 & BHC & 0,677 & 0,153 \\
\hline 26 & BHT & 0,164 & 0,028 \\
\hline 27 & BT6 & 0,407 & 0,126 \\
\hline 28 & BTS & 0,880 & 0,188 \\
\hline 29 & CCM & 1,095 & 0,554 \\
\hline 30 & CYC & 0,788 & 0,239 \\
\hline 31 & DAC & 1,027 & 0,559 \\
\hline 32 & DTC & 0,816 & 0,161 \\
\hline 33 & GMX & 0,958 & 0,594 \\
\hline 34 & HCC & 1,022 & 0,534 \\
\hline 35 & HHL & 1,787 & 0,692 \\
\hline 36 & HLY & 0,948 & 0,446 \\
\hline 37 & HOM & 0,585 & 0,243 \\
\hline 38 & MCC & 1,205 & 1,088 \\
\hline 39 & MCL & 0,417 & 0,220 \\
\hline 40 & NNC & 0,651 & 0,494 \\
\hline 41 & QNC & 0,939 & 0,105 \\
\hline 42 & SCC & 0,943 & 0,710 \\
\hline 43 & SCJ & 1,390 & 0,703 \\
\hline 44 & SDY & 1,427 & 0,479 \\
\hline 45 & SHN & 3,693 & 1,807 \\
\hline 46 & TBX & 0,493 & 0,248 \\
\hline 47 & TCR & 0,759 & 0,376 \\
\hline 48 & TLT & 1,448 & 0,088 \\
\hline 49 & TMX & 1,559 & 0,568 \\
\hline 50 & TSM & 1,404 & 1,047 \\
\hline 51 & TTC & 0,708 & 0,241 \\
\hline 52 & TXM & 1,013 & 0,377 \\
\hline 53 & VCS & 1,177 & 0,500 \\
\hline 54 & VHL & 0,538 & 0,137 \\
\hline 55 & VIT & 0,541 & 0,126 \\
\hline 56 & VTS & 1,078 & 0,647 \\
\hline 57 & YBC & 1,310 & 0,227 \\
\hline
\end{tabular}

Table 10: Market risk of listed construction material firms $(t=20 \%)$. 
Citation: Huy DTN (2014) Changing Tax Rates Impacts on the Risk Level of Vietnam Construction Material Firms. J Entrepren Organiz Manag 3: 110. doi: 10.4172/2169-026X.1000110

Page 9 of 9

\begin{tabular}{|l|c|c|c|}
\hline Statistic results & Equity beta & $\begin{array}{c}\text { Asset beta } \\
\text { (assume debt beta = 0) }\end{array}$ & Difference \\
\hline MAX & 3,693 & 1,807 & 1,885 \\
\hline MIN & 0,129 & 0,041 & 0,088 \\
\hline MEAN & 1,011 & 0,456 & 0,554 \\
\hline VAR & 0,2839 & 0,1101 & 0,174 \\
\hline \multicolumn{3}{|r|}{ Note: Sample size : 57 firms } \\
\hline
\end{tabular}

Table 11: Tax rate $=25 \%$.

\begin{tabular}{|l|c|c|c|}
\hline Statistic results & Equity beta & $\begin{array}{c}\text { Asset beta } \\
\text { (assume debt beta = 0) }\end{array}$ & Difference \\
\hline MAX & 3,693 & 1,807 & 1,885 \\
\hline MIN & 0,132 & 0,030 & 0,102 \\
\hline MEAN & 0,961 & 0,429 & 0,532 \\
\hline VAR & 0,2959 & 0,0989 & 0,197 \\
\hline \multicolumn{3}{|r|}{ Note: Sample size : 57 firms } \\
\hline
\end{tabular}

Table 12: Tax rate $=28 \%$.

\begin{tabular}{|l|c|c|c|}
\hline Statistic results & Equity beta & $\begin{array}{c}\text { Asset beta } \\
\text { (assume debt beta = 0) }\end{array}$ & Difference \\
\hline MAX & 3,693 & 1,807 & 1,885 \\
\hline MIN & 0,124 & 0,028 & 0,096 \\
\hline MEAN & 0,957 & 0,426 & 0,530 \\
\hline VAR & 0,2977 & 0,0982 & 0,200 \\
\hline \multicolumn{3}{|c|}{ Note: Sample size : 57 firms } \\
\hline
\end{tabular}

Table 13: Tax rate $=20 \%$.

of capital providing channels for both construction material and real estate companies.

Finally, this paper suggests implications for further research

and policy suggestion for the Viet Nam government and relevant organizations, economists and investors from current market conditions.

\section{Implications}

Looking at exhibit 13 , it is noted that comparing to asset beta mean and var results of real estate industry in the period 2007-2009 (0,362 and 0,115 ), asset beta mean of construction material industry group is higher while asset beta var is lower $(0,426$ and 0,09$)$. The risk dispersion in case tax rate down $20 \%$ of the construction material is lower than that of real estate industry.

\section{References}

1. Ajinkya BK, Mahesh (2012) Taxation aspects of Mergers and Acquisitions. Asia-Pacific Tax Bulletin.

2. Aldeweireldt J (2012) Tax Treatment of Employee Stock Options: An International Comparison. Tax Notes International.

3. Beeman RE (2009) A Compendium of Financial Crisis Tax Guidance, Bank Accounting \& Finance.

4. Dutt T,Humphery-Jenner M (2013) Stock Return Volatility, Operating Performance and Stock Returns: International Evidence on Drivers of the "Low Volatility" Anomaly. Journal of Banking and Finance 37: 999-1017.

5. FamaEF, Kenneth FR (2004) The Capital Asset Pricing Model: Theory and Evidence.Journal of Economic Perspectives 18: 25-46.

6. Kaouther F (2012) Financial Markets between Efficiency and Persistence: Empirical Evidence on Daily Data.Asian Journal of Finance and Accounting.

7. Gustavo G, Lyandres E, ZhdanovA (2012) Real Options, Volatility and Stock Returns. Journal of Finance 67: 1499-1536.

8. Hall BLiebman J (2000) The Taxation of Executive Compensation.Tax Policy and the Economy 14: 1-44. 